\title{
Multiple Results to Some Biharmonic Problems
}

\author{
Xingdong Tang and Jihui Zhang \\ Mathematical Sciences, Nanjing Normal University, No. 1 Wenyuan Road, Yadong New District, Nanjing, China
}

Correspondence should be addressed to Xingdong Tang; xdtang202@hotmail.com

Received 5 December 2013; Accepted 30 March 2014; Published 15 April 2014

Academic Editor: Xinan Hao

Copyright (C) $2014 \mathrm{X}$. Tang and J. Zhang. This is an open access article distributed under the Creative Commons Attribution License, which permits unrestricted use, distribution, and reproduction in any medium, provided the original work is properly cited.

We study a nonlinear elliptic problem defined in a bounded domain involving biharmonic operator together with an asymptotically linear term. We establish at least three nontrivial solutions using the topological degree theory and the critical groups.

\section{Introduction}

We consider the following biharmonic problem:

$$
\begin{aligned}
& \Delta^{2} u=f(x, u) \quad \text { in } \Omega, \\
& u=\Delta u=0, \quad \text { on } \partial \Omega,
\end{aligned}
$$

where $\Omega \in \mathbb{R}^{N}(N \geqslant 5)$ is a smooth bounded domain and $f: \Omega \times \mathbb{R} \rightarrow \mathbb{R}$ is of class $C^{1}$ with $f(x, 0)=0$.

In the past decades, biharmonic operators have attracted much attention of many researchers and experts. While $f(x, u)=b\left[(u+1)^{+}-1\right]$, the solutions of (1) characterized the travelling waves in a suspension bridge; see [1].

In 1998, Micheletti and Pistoia [2] considered the following biharmonic problem:

$$
\begin{gathered}
\Delta^{2} u+a^{2} \Delta u=b\left[(u+1)^{+}-1\right], \quad \text { in } \Omega, \\
u=\Delta u=0, \quad \text { on } \partial \Omega,
\end{gathered}
$$

where $a, b$ are constants and $\Omega \subset \mathbb{R}^{n}$ is a bounded smooth domain, and they established multiple results by using a minimax process

Three years later, Zhang [3] considered a more general condition; that is,

$$
\begin{gathered}
\Delta^{2} u+c \Delta u=f(x, u), \quad \text { in } \Omega, \\
u=\Delta u=0, \quad \text { on } \partial \Omega,
\end{gathered}
$$

where $c \in \mathbb{R}$ and $f$ satisfies the subcritical growth; at least one nontrivial solution was obtained.
Since then, a lot of papers dealing with biharmonic problems by the critical point theory sprung up, and so forth $[4,5]$.

At the same time, Leray-Schauder degree as a very wonderful tool was introduced to handle biharmonic problems; see [6-9]. To our best knowledge, there are few papers considered (1) by combining the critical point theory (especially Morse theory) with Leray-Schauder degree.

Our argument was originally developed by Hofer [10] and Zhang [11]. Following Hofer [10] and Zhang [11], there are some papers dealing with second-order elliptic problems, and so forth [12].

Zhang [11] first considered the following second-order elliptic problem:

$$
\begin{gathered}
-\Delta u=g(x, u), \quad \text { in } \Omega, \\
B u=0, \quad \text { on } \partial \Omega,
\end{gathered}
$$

where $B$ denotes Neumann operator or Dirichlet operator. Sub- and sup-solutions methods with critical point theory were used to obtain at least two distinct solutions. Also under subcritical growth condition, Chang [13] proved that if $p_{0}$ is an isolated critical point of $J$, then, for all $q \in \mathbb{N}, C_{q}\left(\widetilde{J}, p_{0}\right)=$ $C_{q}\left(J, p_{0}\right)$ with integral coefficients, where $\widetilde{J}, J$ denote the energy functional under $C_{0}(\bar{\Omega}) \cap C^{1}(\bar{\Omega})$ and $H_{0}^{1}(\Omega), C_{q}$ means $q$ th critical group corresponding to (4), which inspires us to consider (1).

Bartsch et al. [12] considered (4) and obtained more results in this direction. Then, some other results in this direction were also obtained; see [14]. 
As far as we know, there are few papers concerned with the biharmonic problem (1) using this method; only Qian and Li [5] considered

$$
\begin{gathered}
\Delta^{2} u+c \Delta u=f(x, u), \quad \text { in } \Omega, \\
u=\Delta u=0, \quad \text { on } \partial \Omega ;
\end{gathered}
$$

And they proved that if $u_{0}$ is an isolated critical point of $J$, then, for all $q \in \mathbb{N}, C_{q}\left(\widetilde{J}, u_{0}\right)=C_{q}\left(J, u_{0}\right)$ with integral coefficients, where $\widetilde{J}, J$ denote the energy functional on the space $C_{0}(\bar{\Omega}) \cap C^{1}(\bar{\Omega})$ and $H_{0}^{1}(\Omega) \cap H^{2}(\Omega), C_{q}$ means $q$ th critical group. In our paper, the results in [5] are improved, and some new results are obtained.

Let $0<\lambda_{1}<\lambda_{2} \leqslant \lambda_{3} \leqslant \cdots \leqslant \lambda_{n} \leqslant \cdots$ denote the eigenvalues of $\left(-\Delta, H_{0}^{1}(\Omega)\right)$ (counting with their multiplicity) with corresponding eigenfunctions $e_{1}, e_{2}, e_{3}, \ldots, e_{n}, \ldots$ We may choose $e_{1}>0$ in $\Omega$. Let $\mu_{k}=\lambda_{k}^{2}, k=1,2, \ldots, n, \ldots$, then $\mu_{1}<\mu_{2} \leqslant \mu_{3} \leqslant \cdots \leqslant \mu_{n} \leqslant \cdots$ are eigenvalues of the following biharmonic problem [3] corresponding eigenfunctions $e_{1}, e_{2}, e_{3}, \ldots, e_{n}, \ldots$;

$$
\begin{gathered}
\Delta^{2} u=\mu u, \quad \text { in } \Omega, \\
u=\Delta u=0, \quad \text { on } \partial \Omega .
\end{gathered}
$$

In order to obtain nontrivial solutions, we now assume that the nonlinearity $f$ satisfies the following conditions:

(f1) $f \in C^{1}(\bar{\Omega} \times \mathbb{R}, \mathbb{R}), f(x, u) u \geqslant 0$ for all $(x, u) \in \bar{\Omega} \times \mathbb{R}$, and there exist constant numbers $C>0$ and $\alpha$ with $1<\alpha<(N+4) /(N-4)$, such that

$$
\left|f_{u}^{\prime}(x, u)\right| \leqslant c\left(1+|u|^{\alpha-1}\right) ;
$$

(f2) there exists $i \in \mathbb{N}$ with $\mu_{2 i}<\mu_{2 i+1}$, such that $f_{u}^{\prime}(x, 0)=$ $\mu_{2 i}$, for all $x \in \bar{\Omega}$;

(f3) $\lim \sup _{|t| \rightarrow \infty} f(x, u) / u<\mu_{1}$ uniformly for $x \in \bar{\Omega}$;

(f4) there is some $r>0$ small, such that

$\mu_{2 i} u^{2} \leqslant F(x, u)<\mu_{2 i+1} u^{2}, \quad u \in \mathbb{R},|u| \leqslant r$, a.e. $x \in \Omega$,

where $F(x, u)=\int_{0}^{u} f(x, s) d s$.

The main result of this paper is the following

Theorem 1. Suppose $f$ satisfies (f1)-(f4). Then (1) has at least three solutions.

\section{Preliminaries}

In this section, we first recall some lemmas and preliminaries.

Let $C_{0}(\bar{\Omega}) \cap C^{k}(\bar{\Omega})$ denote the set of $f: \bar{\Omega} \rightarrow \mathbb{R}$ which are $k$-times continuous differentiable in $\bar{\Omega}$ and identically vanishing on $\partial \Omega$ with the norm $\|u\|_{k}=\sum_{i=0}^{k}\left\|u^{(k)}\right\|_{0}$, where $\|u\|_{0}=\max _{x \in \bar{\Omega}} u(x), P_{k}=\left\{u \in C_{0}(\bar{\Omega}) \cap C^{k}(\bar{\Omega}): u(x) \geqslant\right.$ $0, \forall x \in \Omega\}, \forall k \in \mathbb{N}$.
Lemma 2. $P_{2}$ is a solid cone of $C_{0}(\bar{\Omega}) \cap C^{2}(\bar{\Omega})$; that is, $\stackrel{\circ}{P}_{2} \neq \emptyset$.

It is well known that the positive cone $P_{1}$ is a solid cone of $C_{0}(\bar{\Omega}) \cap C^{1}(\bar{\Omega})$. Our proof depends on the fact above; what is more, the technique we used here is originated from $[15$, page 628].

Proof. Since $P_{1}$ is a closed positive cone of $\left(C_{0}(\bar{\Omega}) \cap C^{1}(\bar{\Omega})\right.$, $\left.\|\cdot\|_{1}\right)$, by the definition of $\|\cdot\|_{k}, k=0,1,2, \ldots, n, \ldots$, for $u \in$ $C_{0}(\bar{\Omega}) \cap C^{2}(\bar{\Omega}),\|u\|_{1} \leqslant\|u\|_{2}$, thus the embedding $i:\left(C_{0}(\bar{\Omega}) \cap\right.$ $\left.C^{2}(\bar{\Omega}),\|\cdot\|_{2}\right) \hookrightarrow\left(C_{0}(\bar{\Omega}) \cap C^{1}(\bar{\Omega}),\|\cdot\|_{1}\right)$ is continuous. $i^{-1}\left(P_{1}\right)$ is closed in $\left(C_{0}(\bar{\Omega}) \cap C^{2}(\bar{\Omega}),\|\cdot\|_{2}\right)$ (in fact $P_{2}=i^{-1}\left(P_{1}\right)$ ). Obviously $\stackrel{\circ}{P}_{1} \cap\left(C_{0}(\bar{\Omega}) \cap C^{2}(\bar{\Omega}),\|\cdot\|_{2}\right) \neq \emptyset$; thus $P_{2}=i^{-1}\left(P_{1}\right)$ has nonempty interior. The proof is finished.

Remark 3. Using the method above, it is not difficult to know that $P_{k}$ is a solid cone in $\left(C_{0}(\bar{\Omega}) \cap C^{k}(\bar{\Omega}),\|\cdot\|_{k}\right), k=$ $2,3,4, \ldots, n, \ldots$..

Remark 4. For any $u \in C_{0}(\bar{\Omega}) \cap C^{2}(\bar{\Omega})$, if $u$ is an interior point of $P_{1}$ in $\left(C_{0}(\bar{\Omega}) \cap C^{1}(\bar{\Omega}),\|\cdot\|_{1}\right)$, then $u$ is an interior point of $P_{2}$ in $\left(C_{0}(\bar{\Omega}) \cap C^{2}(\bar{\Omega}),\|\cdot\|_{2}\right)$.

In what follows, we will use the Hilbert space $V=$ $H_{0}^{1}(\Omega) \cap H^{2}(\Omega)$, and the norm on $V$ is given by $\|u\|_{V}=$ $\int_{\Omega}|\Delta u|^{2} d x$. It is well known that solutions of (1) are critical points of the functional

$$
\Psi(u)=\frac{1}{2} \int_{\Omega}|\Delta u|^{2} d x-\int_{\Omega} F(x, u) d x,
$$

where $F(x, u)=\int_{0}^{u} f(x, s) d s$. Since $f \in C^{1}(\bar{\Omega} \times \mathbb{R}, \mathbb{R})$, it is easy to know that $\Psi \in C^{2}(V, \mathbb{R})$, and

$$
\begin{aligned}
\left\langle\Psi^{\prime}(u), v\right\rangle & =\int_{\Omega}[\Delta u \Delta v-f(x, u) v] d x, \\
\left\langle\Psi^{\prime \prime}(u) v, h\right\rangle & =\int_{\Omega}\left[\Delta v \Delta h-f^{\prime}(x, u) v h\right] d x .
\end{aligned}
$$

Corresponding to the eigenvalues $\mu_{j}^{\prime} s$ we have the splitting $V=H^{-} \oplus N \oplus H^{+}$where

$$
H^{-}=\bigoplus_{j=1}^{2 i-1} e_{j}, \quad N=\operatorname{span}\left\{e_{2 i}\right\}, \quad H^{+}=\overline{\bigoplus_{j=2 i+1}^{+\infty} e_{j}}
$$

Consider the problem

$$
\begin{gathered}
\Delta^{2} u=h, \quad \text { in } \Omega, \\
u=\Delta u=0, \quad \text { on } \partial \Omega .
\end{gathered}
$$

For all $r \in \mathbb{R}^{+}$, denote $B(0, r) \triangleq\left\{u \in C_{0}(\bar{\Omega}) \cap C^{2}(\bar{\Omega})\right.$ : $\left.\|u\|_{2}<r\right\}, U(0, r) \triangleq\left\{u \in V:\|u\|_{V}<r\right\}, P_{2}^{r}=P_{2} \cap B(0, r)$, $\partial P_{2}^{r}=P_{2} \cap \partial B(0, r)$.

Let $K$ denote the solution operator of $(12)$, and $(\mathbf{f} u)(x) \triangleq$ $f(x, u(x))$. Under condition (f1), it is easy to see that $A \triangleq K \mathbf{f}$ : $V \rightarrow V$ is of class $C^{1}$. Since $\mathbf{f}: V \rightarrow V^{*}$ is completely continuous [3], then $A: V \rightarrow V$ is completely continuous. 
Lemma 5 (see $[16]$ ). Suppose $h \in L^{q}(\Omega), q \geqslant 2$; then the weak solution $u=K(h)$ of $(12)$ satisfies $\|u\|_{W^{4, q}} \leqslant C\|h\|_{L^{q}}$; what is more, we have that

$$
K: L^{q}(\Omega) \longmapsto W^{4, q}(\Omega) \cap W_{0}^{1, q}(\Omega)
$$

is continuous.

Remark 6. Actually, for all $h \in V^{*}$, there exists a unique weak solution $u=K(h) \in V$ of (12). Since by Riesz representation theorem, for all $h \in V^{*}$, there exists a unique $\Theta=\Theta(h)$ such that $\langle h, v\rangle=(\Theta, v) \forall v \in V$; thus $\Theta=K(h)$ is the corresponding weak solution.

Consider the Cauchy problem in $V$,

$$
\begin{gathered}
\frac{d}{d t} u(t)=-u(t)+K \mathbf{f} u(t), \\
u(0)=u_{0} .
\end{gathered}
$$

Lemma 7 (see [13]). Let $H$ be a real Hilbert space, and let $\psi \in$ $C^{2}(H, \mathbb{R})$ satisfy the $(P S)$ condition. Assume that

$$
\psi^{\prime}(v)=v-A v, \quad v \in H
$$

where $A$ is a compact mapping, and that $p_{0}$ is an isolated critical point of $f$. Then we have

$$
\operatorname{ind}\left(\psi^{\prime}, p_{0}\right)=\sum_{q=0}^{\infty}(-1)^{q} \operatorname{rank} C_{q}\left(\psi, p_{0}\right) \text {. }
$$

Let $X$ be a retract of a real Banach space $E$, let $U$ be a relatively open subset of $X$, and let $A: \bar{U}_{X} \rightarrow X$ be a completely continuous operator. Suppose that $A$ has no fixed points on $\partial_{X} U$ and that the fixed point of $A$ is bounded. The following lemma establishes the relationship of fixed point index and topological degree.

Lemma 8 (see [17]). If any fixed point of in $U$ is an interior point of $X$, then there exists an open subset $O$ of $E$ with $O \subset U$ such that $O$ contains all fixed points of $A$ in $U$ and

$$
\operatorname{deg}(I-A, O, 0)=i(A, U, X) .
$$

Remark 9. Let $O$ be a bounded open subset of $U$, and let there be no zero points of $I-A$ on $\partial O$. Since $C_{0}(\bar{\Omega}) \cap C^{2}(\bar{\Omega})$ can be compactly embedded into $V$, it follows from the bootstrap argument and the definition of Leray-Schauder degree that

$$
\operatorname{deg}_{V}(I-A, O, 0)=\operatorname{deg}_{C_{0}(\bar{\Omega}) \cap C^{2}(\bar{\Omega})}(I-A, O, 0) .
$$

In what follows, $\operatorname{deg}_{C_{0}^{2}(\bar{\Omega})}$ is denoted simply by deg.

Remark 10 (see [18]). Remark 9 implies that two topological degrees in both $\operatorname{deg}_{C_{0}^{2}(\bar{\Omega})}$ and $\operatorname{deg}_{V}$ are the same. Combining with Lemma 7 , we can obtain the connection between the topological degree and the critical group:

$$
\operatorname{deg}(I-A, O, 0)=\sum_{q=0}^{\infty}(-1)^{q} \operatorname{rank} C_{q}\left(J, p_{0}\right)
$$

Lemma 11. Let $u\left(t, u_{0}\right)$ be the unique solution of (14) with the maximal interval $\left[0, \eta\left(u_{0}\right)\right)$. We have the following conclusions.

(i) If $u_{0} \in C_{0}(\bar{\Omega}) \cap C^{2}(\bar{\Omega})$, then $\left\{u\left(t, u_{0}\right): 0 \leqslant t<\eta\left(u_{0}\right)\right\} \subset$ $C_{0}(\bar{\Omega}) \cap C^{2}(\bar{\Omega})$, and $u\left(t, u_{0}\right)$ is continuous as a function oft from $\left[0, \eta\left(u_{0}\right)\right)$ to $C_{0}(\bar{\Omega}) \cap C^{2}(\bar{\Omega})$.

(ii) If $u_{0}, u^{\star} \in C_{0}(\bar{\Omega}) \cap C^{2}(\bar{\Omega}), u^{\star}=K \mathbf{f} u^{\star}$, and $\| u\left(t, u_{0}\right)-$ $u^{\star} \|_{2} \rightarrow 0$ as $t \rightarrow \eta\left(u_{0}\right)$, then $\left\|u\left(t, u_{0}\right)-u^{\star}\right\|_{2} \rightarrow 0$ ast $\rightarrow \eta\left(u_{0}\right)$.

(iii) If $u_{0} \in C_{0}(\bar{\Omega}) \cap C^{2, \mu}(\bar{\Omega})$ for some $\mu \in(0,1)$ then $\left\{u\left(t, u_{0}\right): 0 \leqslant t<\eta\left(u_{0}\right)\right\} \subset C_{0}(\bar{\Omega}) \cap C^{2 \mu}(\bar{\Omega})$ and is bounded in the $C_{0}^{2, \mu}$ norm.

Lemma 11 essentially comes from [19].

Proof. We only need to construct the embedding chains like (5) and (6) of [19]; the rest can be proved similar to [19, Lemma 2].

Without loss of generality, $\alpha$ can be assumed to satisfy $\max \{8 /(N-4), 1\}<\alpha<(N+4) /(N-4)$. We can choose $\delta>0$, such that

$$
\alpha<\delta+\frac{(N+4)(1-\delta)}{N-4}
$$

Let $q_{0}^{\prime}=2 N /(N-4)$, and define $q_{i}$ by

$$
\frac{1}{q_{i+1}^{\prime}}=\frac{\alpha}{q_{i}^{\prime}}-\frac{2}{N}, \quad i=0,1,3, \ldots
$$

A direct computation shows that

$$
q_{n}^{\prime} \geqslant\left(\frac{5}{5-4 \delta}\right)^{n} q_{0}^{\prime}
$$

Hence there exists a number $n \geqslant 3$ such that

$$
q_{0}^{\prime}<q_{1}^{\prime}<\cdots<q_{n-3}^{\prime}<\frac{N \alpha}{2} \leqslant q_{n-2}^{\prime} .
$$

Let

$$
q_{i}=q_{i}^{\prime}, \quad i=0,1,2, \ldots, n-3,
$$

and choose $q_{n-2}$ and $q_{n-1}$ such that

$$
q_{n-3}<q_{n-2}<\frac{N \alpha}{2}, \quad q_{n-1}=\alpha N .
$$

Let

$$
p_{i}=\frac{q_{i}}{\alpha}, \quad i=0,1,2, \ldots, n-1
$$

Define

$$
\begin{gathered}
X_{0}=L^{q_{0}}(\Omega), \quad X_{i+1}=W^{4, p_{i}}(\Omega) \cap W_{0}^{1, p_{i}}, \\
Y_{i}=L^{p_{i}}(\Omega), \quad Z_{i}=L^{q_{i}}(\Omega), \\
i=0,1, \ldots, n-1 .
\end{gathered}
$$

Then we have the following imbedding chains: 


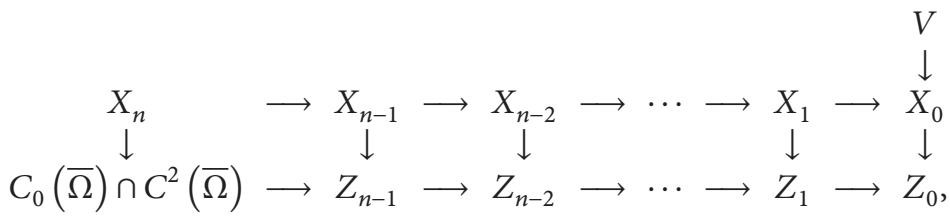

$$
\begin{aligned}
& Y_{n-1} \longrightarrow Y_{n-2} \longrightarrow \cdots \longrightarrow Y_{1} \longrightarrow Y_{0} .
\end{aligned}
$$

What is more, we have the chains of bounded and continuous operators

$$
Z_{i} \stackrel{\mathrm{f}}{\rightarrow} Y_{i} \stackrel{K}{\rightarrow} X_{i+1}, \quad i=0,1,2, \ldots, n-1
$$

Lemma 12. Suppose that ( $f 1)$ and $(f 3)$ hold. Then $\Psi$ satisfies the (PS) condition.

The proof of this lemma is similar to the proof of [5, Lemma 2.1]. We omit it here.

Since $A: V \rightarrow V$ is completely continuous, then by the above bootstrap iteration, $A: C_{0}(\bar{\Omega}) \cap C^{2}(\bar{\Omega}) \rightarrow$ $C_{0}(\bar{\Omega}) \cap C^{2}(\bar{\Omega})$ is completely continuous. For our application, sometimes we would consider the restriction $\widetilde{\Psi}$ of $\Psi$ on a smaller Banach space $C_{0}(\bar{\Omega}) \cap C^{2}(\bar{\Omega})$. The functional may lose the (PS) condition. However following [20], the following two lemmas can be obtained.

Lemma 13 (see [20]). Suppose that (f1) and (f3) hold. Then $\widetilde{\Psi}$ possesses the following properties.

(i) $\widetilde{\Psi}(K)$ is a closed subset.

(ii) For each pair $a<b, K \cap \widetilde{\Psi}^{-1}(a, b)=\emptyset$ implies that $\widetilde{\Psi}_{a}$ is a strong deformation retract of $\widetilde{\Psi}_{b} \backslash K_{b}$, where $K$ denotes the critical set of $\Psi$ (and also $\widetilde{\Psi}$ ).

Lemma 14 (see [20]). $C_{*}\left(\widetilde{\Psi}, p_{0}\right)=C_{*}\left(\Psi, p_{0}\right)$ with integral coefficients.

Here and in what follows, we always assume that $\Psi$ has only finitely many critical points.

Lemma 15 (see [21]). Let 0 be an isolated critical point of $\Psi \in$ $C^{2}(E, \mathbb{R})$, where $N=\operatorname{ker}\left[\Psi^{\prime}(0)\right]$. Denote $\mu=\operatorname{dim} E^{-}<\infty$, $v=\operatorname{dim} N<\infty$, and assume that $\Psi$ has a local linking at 0 with respect to a direct sum decomposition $E=W^{-} \oplus W^{+}$, where $W^{-}=E^{-} \oplus N$; that is, there exists $r>0$ small such that

$$
\begin{array}{ll}
\Psi(u)>0 & \text { for } u \in W_{+}, 0<\|u\|_{V} \leqslant r, \\
\Psi(u) \leqslant 0 & \text { for } u \in W_{-},\|u\|_{V} \leqslant r .
\end{array}
$$

Then

$$
C_{q}(\Psi, 0)=\delta_{q, k} \mathbb{F} \quad \text { for } k=\mu+\nu
$$

\section{Calculation of Degree}

Lemma 16. Suppose that (f1), (f3), and (f4) hold. Then there exists $r_{l k}>0$, such that, for all $r \in\left(0, r_{l k}\right]$,

$$
\operatorname{deg}(I-A, B(0, r), 0)=0 .
$$

Proof. Since $\left\{e_{j}\right\}_{j=1}^{\infty}$ is an orthogonal basis of $V$, for $u \in V$, there exist $\left\{a_{j}\right\}_{j=1}^{\infty} \subset \mathbb{R}$, such that $u=\sum_{j=1}^{\infty} a_{j} e_{j}$. Let

$$
E^{-}=H^{-} \oplus N, \quad E^{+}=H^{+} .
$$

Since $E^{-}$is finite dimensional, we have that, for given $r>0$, there exists some $\rho>0$ such that if

$$
u \in E^{-}, \quad\|u\|_{V} \leqslant \rho,
$$

then

$$
|u(x)| \leqslant \frac{r}{3}<r, \quad \text { a.e. } x \in \Omega .
$$

By (f4), for $u \in E^{-}$with $\|u\| \leqslant \rho$,

$$
\begin{aligned}
\Psi(u) & =\frac{1}{2} \int_{\Omega}\left(|\Delta u|^{2}\right) d x-\int_{\Omega} F(x, u) d x \\
& \leqslant \frac{1}{2} \int_{\Omega}\left(|\Delta u|^{2}-\mu_{2 i} u^{2}\right) d x \\
& =\frac{1}{2} \int_{\Omega}\left(\left|\Delta \sum_{j=1}^{2 i} a_{j} e_{j}\right|^{2}-\mu_{2 i} \sum_{j=1}^{2 i} a_{j} e_{j}\right) d x \\
& \leqslant \frac{1}{2} \int_{\Omega}\left(|\Delta u|^{2}-m u_{2 i} u^{2}\right) d x \\
& =\frac{1}{2} \int_{\Omega}\left(\left|\sum_{j=1}^{2 i} \mu_{j} a_{j} e_{j}\right|^{2}-\sum_{j=1}^{2 i} \mu_{2 i} a_{j} e_{j}\right) d x \leqslant 0 .
\end{aligned}
$$

For $u \in E^{+}$with $0<\|u\| \leqslant \rho$,

$$
\begin{aligned}
\Psi(u) & =\frac{1}{2} \int_{\Omega}\left(|\Delta u|^{2}\right) d x-\int_{\Omega} F(x, u) d x \\
& >\frac{1}{2} \int_{\Omega}\left(|\Delta u|^{2}-\mu_{2 i+1} u^{2}\right) d x \\
& =\frac{1}{2} \int_{\Omega}\left(\left|\Delta \sum_{j=2 i+1}^{\infty} a_{j} e_{j}\right|^{2}-m u_{2 i+1} \sum_{j=2 i+1}^{\infty} a_{j} e_{j}\right) d x \\
& =\frac{1}{2} \int_{\Omega}\left(\left|\sum_{j=2 i}^{\infty} \mu_{j} a_{j} e_{j}\right|^{2}-\sum_{j=2 i+1}^{\infty} \mu_{2 i+1} a_{j} e_{j}\right) d x \geqslant 0 .
\end{aligned}
$$


Thus $\Psi$ possesses a local linking at the origin. By Lemma 15, the critical groups of $\Psi$ at the origin satisfy

$$
C_{\mu_{2 i}}(\Psi, 0) \neq 0 \text {. }
$$

Then there exists $r_{l k}>0$ small such that there is no other critical point in $B\left(0, r_{l k}\right)$ except 0 , for all $r \in\left(0, r_{l k}\right]$, and the following can be obtained by Lemma 13 and Remark 10:

$$
\operatorname{deg}(I-A, B(0, r), 0)=1 .
$$

Lemma 17. Suppose ( f1) and ( $f 2)$ hold. There exists $r_{1} \in\left(0, r_{l k}\right]$ ( $r_{l k}$ is defined in Lemma 16), such that, for all $r \in\left(0, r_{1}\right]$,

$$
i\left(A, P_{2}^{r}, P_{2}\right)=0, \quad i\left(A,-P_{2}^{r},-P_{2}\right)=0 .
$$

Proof. We only prove that $i\left(A, P_{2}^{r}, P_{2}\right)=0$. By (f1), $A\left(P_{2}\right) \subset P_{2}$, it follows from the condition (f2) that there exist $\delta_{1}>0$ and $\rho_{1} \in\left(0, r_{l k}\right]$ such that

$$
f(x, t) \geqslant \mu_{1}\left(1+\delta_{1}\right) t, \quad(x, t) \in \bar{\Omega} \times\left[0, \rho_{1}\right] .
$$

If $u=A u+v e_{1}$ for some $v \geqslant 0$ and $u \in \partial P_{2}^{r}$, where $r \in\left(0, \rho_{1}\right]$ is a positive number, that is

$$
\begin{gathered}
\Delta^{2} u=f(x, u)+v e_{1}, \quad \text { in } \Omega . \\
u=\Delta u=0, \quad \text { on } \partial \Omega,
\end{gathered}
$$

then we have from (41) that

$$
\begin{gathered}
\Delta^{2} u \geqslant \mu_{1}\left(1+\delta_{1}\right) u, \quad \text { in } \Omega . \\
u=\Delta u=0, \quad \text { on } \partial \Omega .
\end{gathered}
$$

Thus,

$$
\mu_{1} \int_{\Omega} u(x) e_{1}(x) d x \geqslant \mu_{1}\left(1+\delta_{1}\right) \int_{\Omega} u(x) e_{1}(x) d x
$$

and this is a contradiction. Therefore, according to the property of fixed point index, we get

$$
i\left(A, P_{2}^{r}, P_{2}\right)=0 .
$$

Similarly, we can also show that there exists $\rho_{2} \in\left(0, r_{0}\right]$ such that $i\left(A,-P_{2}^{r},-P_{2}\right)=0$ for all $r \in\left(0, \rho_{2}\right]$. Let $r_{1}=$ $\min \left\{\rho_{1}, \rho_{2}\right\}$. Then the conclusion holds.

Lemma 18. Suppose that (f1) and (f3) hold. Then there exists $\mathrm{O}_{+} \subset P_{2} \backslash\{0\}, O_{-} \subset\left(-P_{2} \backslash\{0\}\right)$, such that

$$
\operatorname{deg}\left(I-A, O_{+}, 0\right)=1, \quad \operatorname{deg}\left(I-A, O_{-}, 0\right)=1 .
$$

Proof. Since $\lim _{|t| \rightarrow \infty} f(x, t) / t<\mu_{1}$ uniformly for $x \in \bar{\Omega}$, there exist constants $\delta \in(0,1)$ and $C_{1}>0$ such that

$$
f(x, t) \leqslant \mu_{1}(1-\delta) t+C_{1}, \quad(x, t) \in \bar{\Omega} \times[0, \infty) .
$$

We will first show that any solution of (1) is bounded. Suppose $u_{0}$ is a solution; then $u_{0}$ satisfy

$$
\begin{gathered}
\Delta^{2} u_{0}=f\left(x, u_{0}\right), \quad \text { in } \Omega, \\
u_{0}=\Delta u_{0}=0, \quad \text { on } \partial \Omega .
\end{gathered}
$$

Multiplying by $u_{0}$, we have

$$
\left\|u_{0}\right\|_{V}^{2}=\int_{\Omega} f\left(x, u_{0}\right) u_{0}, \quad x \in \Omega
$$

by (47), it is easy to see

$$
\left\|u_{0}\right\|_{V}^{2}=\int_{\Omega} \mu_{1}(1-\delta) u_{0}^{2}+\int_{\Omega} C_{1} u_{0}, \quad x \in \Omega .
$$

Let $\epsilon<\mu_{1} \delta /\left(2 C_{1}\right)$, and using Young inequality, there exists constant $C_{2}=C_{2}\left(\delta, C_{1}\right)$ such that

$$
\left\|u_{0}\right\|_{V}^{2} \leqslant \int_{\Omega} \mu_{1}\left(1-\frac{\delta}{2}\right) u_{0}^{2}+C_{2}|\Omega|^{1 / 2} .
$$

By Poincaré inequality, there exists $C_{3}>0$ only dependent on $\Omega$, such that

$$
\left\|u_{0}\right\|_{V}<C_{3}
$$

By a bootstrap argument, there exists $R_{1}>0$ such that $\left\|u_{0}\right\|_{2}<$ $R_{1}$.

Let $R>R_{1}$, and we will show that

$$
A u \neq v u, \quad \forall x \in P_{2} \cap \partial B(0, R), \quad v \geqslant 1 .
$$

Suppose there exists $v_{0} \geqslant 1,\left\|u_{0}\right\|_{V}=R$, such that $A u_{0}=v_{0} u_{0}$; then $v_{0}>1$. By (f3), such that $f(x, t) \leqslant \mu_{1}(1-\delta) t+C_{1}$, for all $t \geqslant 0$,

$$
v_{0} u_{0}=A u_{0} \leqslant \mu_{1}(1-\delta) K u_{0}+C_{4} K 1
$$

Then $\left[1-\mu_{1}(1-\delta) / K\right] u_{0} \leqslant C_{5}$, where $C_{5}=$ $C_{1}\|K\|_{L\left(C_{0}(\bar{\Omega}) \cap C^{2}(\bar{\Omega}), C_{0}(\bar{\Omega}) \cap C^{2}(\bar{\Omega})\right)}$. Then $u_{0} \leqslant C_{6}$, where $C_{6}=$ $\left\|(I-K)^{-1}\right\| C_{5}$. Let $R=C_{6}$; then, for all $u \in \partial B(0, R)$, we have

$$
A u \neq v u \quad \forall x \in \partial P_{2}^{R}, v \geqslant 1 .
$$

Then

$$
i\left(A, P_{2}^{R}, P_{2}\right)=1 \text {. }
$$

From Lemma 17, we have

$$
i\left(A, P_{2}^{R} \backslash \overline{P_{2}^{r}}, P_{2}\right)=1 .
$$

By Lemma 18 and the strong maximum principle of second order elliptic problem, it is easy to know that if $u \in P_{2} \backslash\{0\}$ is a solution of (1), then $u \in \stackrel{\circ}{P}_{2}$, and thus, by Lemma 2, $u \in \stackrel{\circ}{P}_{2}$. Using Lemma 8 , there is a bounded open $O_{1} \subset P_{2} \cap$ $(B(0, R) \backslash B(0, r))$, such that

$$
\operatorname{deg}\left(I-A, O_{+}, 0\right)=1
$$

Similarly, there is a bounded open subset $O_{-} \subset-\left(P_{2}^{R} \backslash \bar{P}_{2}^{r}\right)$, such that

$$
\operatorname{deg}\left(I-A, O_{-}, 0\right)=1
$$




\section{Proof of Main Result}

Proof. By conditions (f1) and (f3), it is easy to know that

$$
\operatorname{ind}(I-A, \infty)=\operatorname{ind}\left(I-A^{\prime}(\infty), 0\right)=(-1)^{0}=1 \text {; }
$$

that is, there exists $\bar{R}>R$ large enough, such that

$$
\operatorname{deg}(I-A, B(0, \bar{R}), 0)=\operatorname{ind}(I-A, \infty)=1 .
$$

If $A$ has no fixed point in $B(0, \bar{R}) \backslash\left(P_{2}^{R} \cup\left(-P_{2}^{R}\right)\right)$, then the additivity property of degree implies

$$
\begin{aligned}
\operatorname{deg}(I-A, B(0, \bar{R}), 0) \\
=\operatorname{deg}\left(I-A, O_{+}, 0\right) \\
\quad+\operatorname{deg}\left(I-A, O_{-}, 0\right)+\operatorname{deg}\left(I-A, B\left(0, \frac{r}{2}\right), 0\right) .
\end{aligned}
$$

It follows that $1=1+1+1$. This is a contradiction. Thus (1) has at least a solution $u_{3}$ in $B(0, \bar{R}) \backslash\left(P_{2}^{R} \cup\left(-P_{2}^{R}\right) \cup B(0,(r / 2))\right)$.

\section{Conflict of Interests}

The authors declare that there is no conflict of interests regarding the publication of this paper.

\section{Acknowledgments}

This research was supported by the National Natural Science Foundation of China (no. 10871096) and the Project of Graduate Education Innovation of Jiangsu Province (CXLX13_367).

\section{References}

[1] P. J. McKenna and W. Walter, "Nonlinear oscillations in a suspension bridge," Archive for Rational Mechanics and Analysis, vol. 98, no. 2, pp. 167-177, 1987.

[2] A. M. Micheletti and A. Pistoia, "Nontrivial solutions for some fourth order semilinear elliptic problems," Nonlinear Analysis: Theory, Methods \& Applications, vol. 34, no. 4, pp. 509-523, 1998.

[3] J. Zhang, "Existence results for some fourth-order nonlinear elliptic problems," Nonlinear Analysis: Theory, Methods \& Applications, vol. 45, no. 1, pp. 29-36, 2001.

[4] S. T. Kyritsi and N. S. Papageorgiou, "On superquadratic periodic systems with indefinite linear part," Nonlinear Analysis: Theory, Methods \& Applications, vol. 72, no. 2, pp. 946-954, 2010.

[5] A. X. Qian and S. J. Li, "Multiple solutions for a fourthorder asymptotically linear elliptic problem," Acta Mathematica Sinica, vol. 22, no. 4, pp. 1121-1126, 2006.

[6] R. Ma and H. Wang, "On the existence of positive solutions of fourth-order ordinary differential equations," Applicable Analysis, vol. 59, no. 1-4, pp. 225-231, 1995.

[7] Z. Wei and C. Pang, "Positive solutions and multiplicity of fourth-order $m$-point boundary value problems with two parameters," Nonlinear Analysis: Theory, Methods \& Applications, vol. 67, no. 5, pp. 1586-1598, 2007.
[8] J. Xu and Z. Yang, "Positive solutions for a fourth order $p$ Laplacian boundary value problem," Nonlinear Analysis: Theory, Methods \& Applications, vol. 74, no. 7, pp. 2612-2623, 2011.

[9] M. Zhang and Z. Wei, "Existence of positive solutions for fourth-order $m$-point boundary value problem with variable parameters," Applied Mathematics and Computation, vol. 190, no. 2, pp. 1417-1431, 2007.

[10] H. Hofer, "Variational and topological methods in partially ordered Hilbert spaces," Mathematische Annalen, vol. 261, no. 4, pp. 493-514, 1982.

[11] G. Q. Zhang, "Variational methods and sub- and supersolutions," Scientia Sinica A: Mathematical, Physical, Astronomical \& Technical Sciences, vol. 26, no. 12, pp. 1256-1265, 1983.

[12] T. Bartsch, K.-C. Chang, and Z.-Q. Wang, "On the Morse indices of sign changing solutions of nonlinear elliptic problems," Mathematische Zeitschrift, vol. 233, no. 4, pp. 655-677, 2000.

[13] K.-C. Chang, Infinite-Dimensional Morse Theory and Multiple Solution Problems, vol. 6 of Progress in Nonlinear Differential Equations and their Applications, Birkhäuser Boston, Boston, Mass, USA, 1993.

[14] Z. Liu and J. Sun, "An elliptic problem with jumping nonlinearities," Nonlinear Analysis; Theory, Methods \& Applications, vol. 63, no. 8, pp. 1070-1082, 2005.

[15] H. Amann, "Fixed point equations and nonlinear eigenvalue problems in ordered banach spaces," SIAM Review, vol. 18, no. 4, pp. 620-709, 1976.

[16] J. Chabrowski and J. Marcos do Ó, "On some fourth-order semilinear elliptic problems in $\mathbb{R}^{n}$," Nonlinear Analysis: Theory, Methods \& Applications, vol. 49, no. 6, pp. 861-884, 2002.

[17] X. Xu, "Multiple sign-changing solutions for some m-point boundary-value problems," Electronic Journal of Differential Equations, vol. 89, pp. 1-14, 2004.

[18] F. Li and Y. Li, "Multiple sign-changing solutions to semilinear elliptic resonant problems," Nonlinear Analysis: Theory, Methods \& Applications, vol. 72, no. 9-10, pp. 3820-3827, 2010.

[19] Z. Liu, "Positive solutions of superlinear elliptic equations," Journal of Functional Analysis, vol. 167, no. 2, pp. 370-398, 1999.

[20] G. Q. Zhang, "A variant mountain pass lemma," Scientia Sinica A: Mathematical, Physical, Astronomical \& Technical Sciences, vol. 26, no. 12, pp. 1241-1255, 1983.

[21] J. Su, "Multiplicity results for asymptotically linear elliptic problems at resonance," Journal of Mathematical Analysis and Applications, vol. 278, no. 2, pp. 397-408, 2003. 


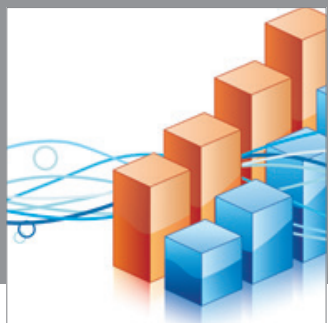

Advances in

Operations Research

mansans

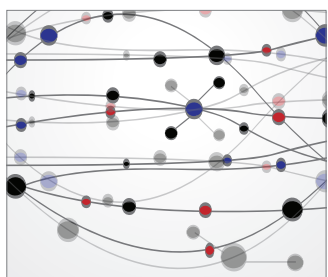

The Scientific World Journal
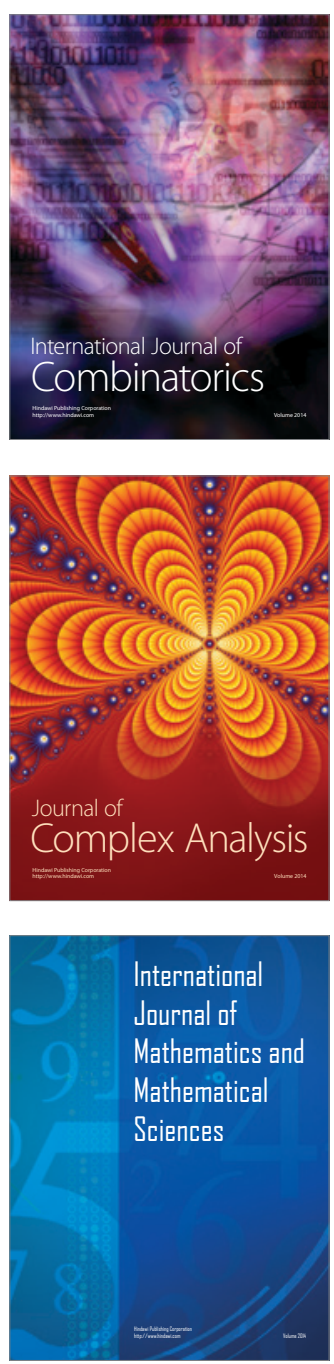
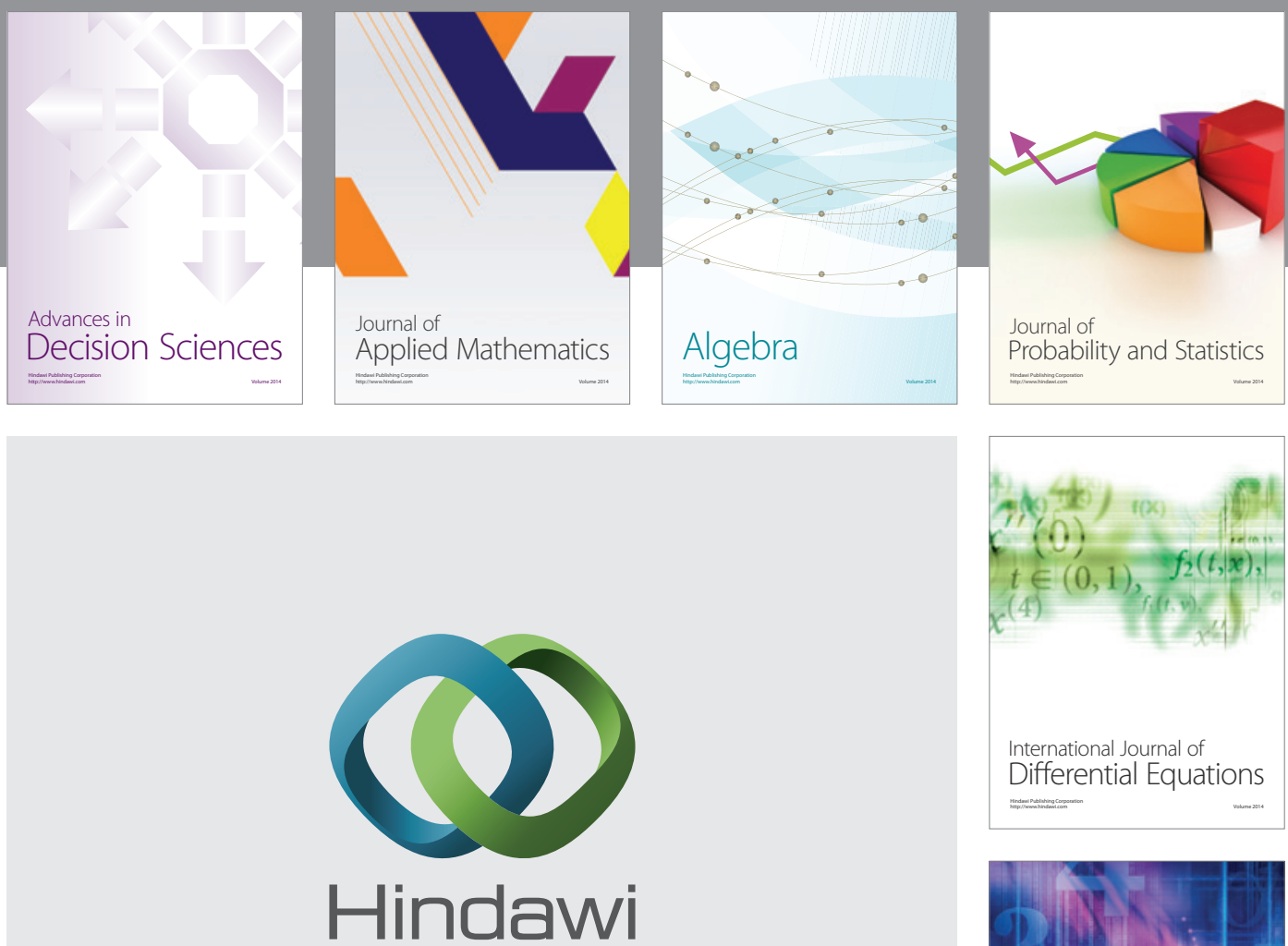

Submit your manuscripts at http://www.hindawi.com
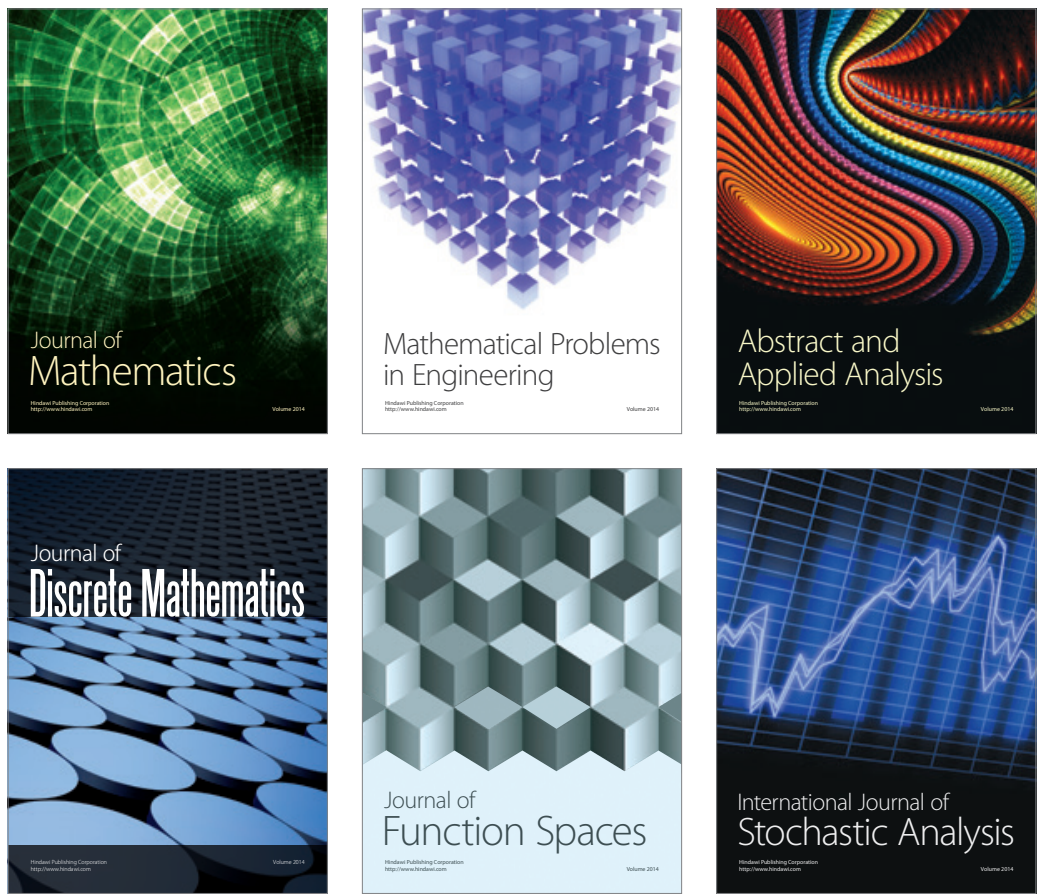

Journal of

Function Spaces

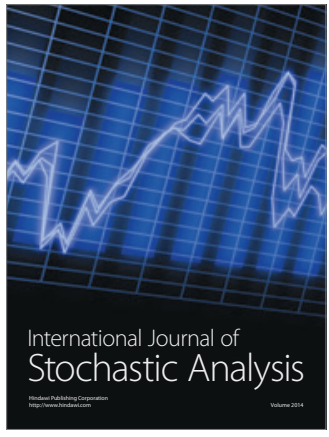

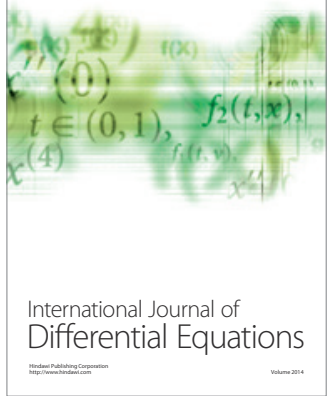
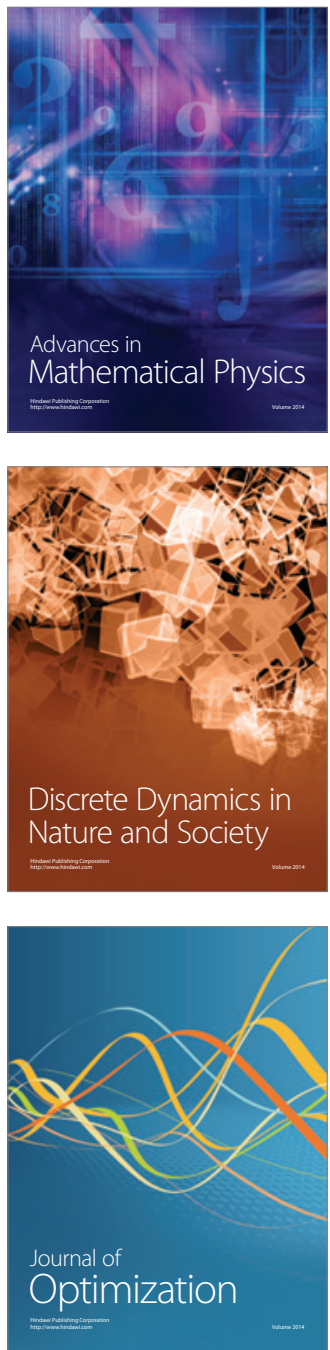